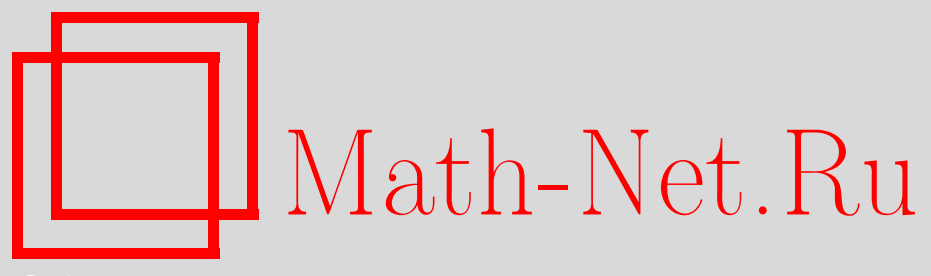

В. П. Маслов, Переход в конденсатное состояние для классических газов и кластеризация, Матем. заметки, 2008, том 84, выпуск 6, 851-873

DOI: https://doi.org/10.4213/mzm6566

Использование Общероссийского математического портала Math-Net.Ru подразумевает, что вы прочитали и согласны с пользовательским соглашением http://www . mathnet.ru/rus/agreement

Параметры загрузки:

IP : 54.80 .97 .219

26 апреля 2023 г., 17:54:16

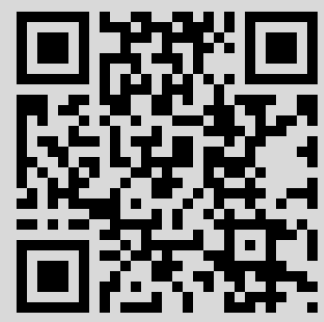


Том 84 выпуск 6 декабрь 2008

УДК $519.2+531.19$

\section{Переход в конденсатное состояние для классических газов и кластеризация}

\section{В. П. Маслов}

В работе на примере строгой формулировки и строгого доказательства распределения Максвелла устанавливаются оценки распределения в зависимости от параметра $N$ - числа частиц. Далее рассматривается задача о возникновении димеров в классическом газе как аналог бозе-конденсации и устанавливаются оценки нижнего уровня аналога бозе-конденсации. Определяется связь этого уровня с теорией "захвата" в задаче рассеяния, отвечающей взаимодействию вида потенциала Леннарда-Джонса. Это решает также проблему парадокса Гиббса.

Приводится вывод уравнения состояний неидеального газа как результат парного взаимодействия частиц в моделях Леннарда-Джонса и обсуждается

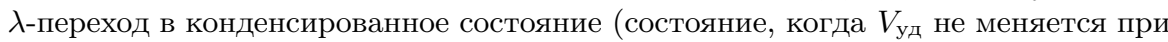
увеличении давления; для теплоемкости - это $\lambda$-точка) для классических газов.

Приводятся также новые квантовые уравнения для протекания нейтрального газа, состоящего из частиц с нечетным числом нейтронов, в капиллярах в модели Сузерленда.

Библиография: 36 названия.

Мы будем рассматривать классический газ.

Распределение Максвелла имеет вид

$$
\mathscr{N}_{d v}=\left(\frac{m}{2 \pi k T}\right)^{3 / 2} e^{-\left(m\left(v_{x}^{2}+v_{y}^{2}+v_{z}^{2}\right)\right) / 2 k T} d v_{x} d v_{y} d v_{z},
$$

где $v$ - скорость, $T$ - температура, $k$ - константа Больцмана, $\mathscr{N}_{d v}$ - относительное число частиц, содержащихся в интервале $d v=d v_{x} d v_{y} d v_{z}$.

Надо иметь в виду, что как плотность распределения в общем случае оно неверно, а верно лишь в коммулятивном варианте. Это значит, что интеграл от плотности (1) по любому ограниченному снизу узкому конечному интервалу скоростей действительно определяет относительное число частиц $\mathscr{N}_{v_{1} v_{2}}$ в этом диапазоне скоростей.

Мы получим обычное распределение Максвелла для достаточно узкого интервала скоростей:

$$
\mathscr{N}_{v_{1} \sqrt{\kappa}, v_{2} \sqrt{\kappa}}=\frac{\int_{\left|v_{1}\right| \sqrt{\kappa}}^{\left|v_{2}\right| \sqrt{\kappa}} e^{-m v^{2} / 2 k T} v^{2} d|v|}{\int_{-\infty}^{\infty} e^{-m v^{2} / 2 k T} v^{2} d|v|}
$$

где $\mathscr{N}_{v_{1} \sqrt{\kappa}, v_{2} \sqrt{\kappa}}$ - относительное число частиц, попадающих в интервал скоростей $\left|v_{1}\right| \sqrt{\kappa},\left|v_{2}\right| \sqrt{\kappa}, \kappa-$ малый параметр.

(C) В. П. МАслов, 2008 
Заменой переменных малый параметр $\kappa$ можно убрать, переведя его в экспоненту, т.e.

$$
\mathscr{N}_{v_{1} v_{2}}=\frac{\int_{\left|v_{1}\right|}^{\left|v_{2}\right|} e^{-\left(\kappa m v^{2}\right) / 2 k T} v^{2} d|v|}{\int_{-\infty}^{\infty} e^{-\left(\kappa m v^{2}\right) / 2 k T} v^{2} d|v|}, \quad \text { при } \quad \kappa \rightarrow 0 .
$$

Эту формулу как асимптотическую формулу мы получим ниже и получим оценку, т.е. область ее применимости.

Рассмотрим наиболее часто употребляемый потенциал взаимодействия Леннарда-Джонса:

$$
\varphi(r)=4 U_{0}\left[\left(\frac{\sigma}{r}\right)^{12}-\left(\frac{\sigma}{r}\right)^{6}\right],
$$

где величина $\sigma$ означает расстояние, на котором потенциальная функция меняет знак, а $U_{0}$ - минимальное значение потенциала (в точке $r=2^{1 / 6} \sigma$ ) или глубину потенциальной ямы.

Чтобы соблюсти размерность в выражении (1), из тех величин, которые участвуют в определении данной частицы (нейтральной молекулы), мы можем для данной молекулы записать

$$
\left(\frac{m}{U_{0}}\right)^{3 / 2} \int_{-\infty}^{\infty} e^{-\left(\kappa m v^{2}\right) / 2 k T} d v_{x} d v_{y} d v_{z}=N
$$

Отсюда

$$
\frac{4}{3} \cdot \frac{\pi}{\kappa^{3 / 2}}\left(\frac{2 k T}{U_{0}}\right)^{3 / 2}=N
$$

т.e.

$$
\kappa=\left(\frac{2 k T}{U_{0}}\right) N^{-2 / 3} \cdot\left(\frac{4 \pi}{3}\right)^{2 / 3} .
$$

Следовательно, интервал скоростей, на котором мы могли бы определить относительное число частиц, равен

$$
\left\{\left(\frac{2 k T}{U_{0}}\right)^{1 / 2}\left|v_{1}\right| N^{-1 / 3},\left(\frac{2 k T}{U_{0}}\right)^{1 / 2}\left|v_{2}\right| N^{-1 / 3}\right\},
$$

где $v_{1}$ и $v_{2}-$ произвольные скорости, $\left|v_{2}\right|>\left|v_{1}\right|$, не зависящие от числа $N$.

Для строгого обоснования распределения Максвелла мы будем использовать то, казалось бы, незначительное обстоятельство, что число частиц $N$ - целое, и в конечном счете применять теорию чисел, которая, казалось бы априори, тут не при чем.

Распределение Максвелла столь же важно в теории сложности, как распределение Пуассона, Гаусса и другие классические распределения.

Определим энергию

$$
E=\frac{4 \pi / 3 \cdot(k T / 2)^{5 / 2}}{U_{0}^{3 / 2}} .
$$

Распределение в диапазоне энергий между $m v_{1}^{2} / 2$ и $m v_{2}^{2} / 2$ должно иметь следующий вид:

$$
\frac{E_{v_{1} v_{2}}}{E}=\frac{\int_{m v_{1}^{2} / 2}^{m v_{2}^{2} / 2} \xi e^{-\kappa(\xi / k T)} d \xi^{3 / 2}}{\int_{0}^{\infty} \xi e^{-\kappa(\xi / k T)} d \xi^{3 / 2}}=\frac{\int_{\kappa\left(m v_{1}^{2} / 2\right)}^{\kappa\left(m v^{2} / 2\right)} \xi e^{-(\xi / k T)} d \xi^{3 / 2}}{\int_{0}^{\infty} \xi e^{-(\xi / k T)} d \xi^{3 / 2}}
$$


Не уменьшая общности, положим

$$
\frac{m v_{1}^{2}}{2}=l U_{0}, \quad \frac{m v_{2}^{2}}{2}=(l+1) U_{0},
$$

где $l$ - целое число. Отсюда с точностью до $O\left(\kappa^{2}\right)$

$$
\frac{E_{v_{1} v_{2}}}{E}=\frac{U_{0} \kappa l^{1 / 2} l}{E}+O\left(\kappa^{2}\right) l^{1 / 2} \frac{U_{0}}{E} .
$$

Если разбить полную энергию $E$ на интервалы вида $\left\{l U_{0},(l+1) U_{0}\right\}, l=0,1, \ldots, l_{E}$, так, чтобы сумма этих интервалов была меньше $E$ на величину порядка $O(\kappa)$, то в силу формулы Эйлера-Маклорена будет иметь порядок

$$
l_{E} \cong \kappa^{-7 / 5} \text {. }
$$

Далее, если заменить $l^{1 / 2}$ на целую часть $\left[l^{1 / 2}\right]$, то мы уменьшим сумму интервалов не более, чем на величину $O\left(\kappa^{-7 / 5}\right)$.

Отсюда для объединения разбиений $\kappa \sum_{0}^{l_{E}} U_{0}\left[l^{1 / 2}\right] l$ мы получим неравенство

$$
E-O\left(\kappa^{-7 / 5}\right) \leqslant \kappa U_{0} \sum_{0}^{l_{E}}\left[l^{1 / 2}\right] l \leqslant E .
$$

Мы таким образом получили энергетические ячейки (ящики) и хотим определить наиболее вероятное число частиц, попадающих в каждый ящик.

Теперь разобьем число частиц $N=\sum N_{j k}$, где $k=1,2, \ldots,\left[j^{1 / 2}\right], j=1,2, \ldots, l_{E}$.

Следовательно, при условии (11) мы получим следующее ограничение для нашего разбиения:

$$
E-O\left(\kappa^{-7 / 5}\right) \leqslant \kappa U_{0} \sum_{j=1}^{l_{E}} \sum_{k=1}^{\left[j^{1 / 2}\right]} N_{j k} \leqslant E .
$$

Условие $N=\sum N_{j k}$ означает, что объем упорядоченной выборки с возвращением [1] равен $N$, а условие (12) - что энергия, отвечающая этой выборке, заключена в интервале $\left\{E-O\left(\kappa^{-7 / 5}\right), E\right\}$.

Это были эвристические соображения. Итак, мы сделаем следующие предположения.

Рассматривается система $N$ частиц в объеме $V$, обладающих энергией $E$. При этом $N \rightarrow \infty, E / U_{0} \rightarrow \infty$.

Интервал $(O, E)$ разбивается на малые по сравнению с $E$ подынтервалы $E_{i}<$ $E_{i+1}, i=1, \ldots, l_{0}$, и соответствующие им интервалы модулей скоростей $\left|v_{i}\right|<v_{i+1}$, а также интервалы фазового объема, энергетические ящики $\Delta \Omega_{i}$, которые заключены между этими скоростями

$$
\Delta \Omega_{i}=\int_{\left|v_{i}\right|}^{\left|v_{i+1}\right|} \frac{m v^{2}}{2} d v_{1} d v_{2} d v_{3}=\operatorname{const}\left(E_{i+1}^{5 / 2}-E_{i}^{5 / 2}\right) V \approx \operatorname{const} \Delta E_{i} E_{i}^{3 / 2} V .
$$

В этих энергетических ящиках мы размещаем всевозможными способами $N$ различающихся между собой частиц. То есть совершаем упорядоченную выборку с возвращением из $N$ “шаров" по указанным энергетическим ящикам (фазовым объемам) 
способом, указанным в (12)

$$
0 \leqslant \kappa U_{0} \sum_{j=1}^{l_{E}} \sum_{k=1}^{\left[j^{1 / 2}\right]} N_{j k} \leqslant E .
$$

Мы обозначили через $\mathscr{N}\left(\left|v_{1}\right|,\left|v_{2}\right|\right)$ относительное число частиц в интервале скоростей $\left(v_{1}, v_{2}\right)$.

При перечисленных условиях имеет место следующая теорема.

Теорема 1. Вероятность того, что не выполнена оценка

$$
\begin{aligned}
& \mathscr{N}\left(\left|v_{1}\right|,\left|v_{1}\right|+O\left(N^{-1 / 2+\delta}\right)\right)-\frac{\int_{\left|v_{1}\right|}^{\left|v_{1}\right|+O\left(N^{-1 / 2+\delta}\right)} e^{-m v^{2} / 2 k T} d v_{1} d v_{2} d v_{3}}{\int_{-\infty}^{-\infty} e^{-m v^{2} / 2 k T} d v_{1} d v_{2} d v_{3}} \\
& =O\left(\frac{\sqrt{\ln N}}{\sqrt{N}}|\ln \ln N|^{\varepsilon}\right),
\end{aligned}
$$

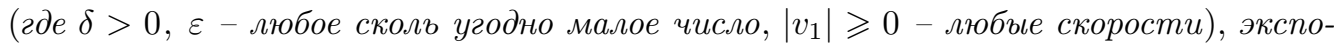
ненциально мала (меньше чем $1 / N^{k}$, где $k$-любое целое).

Это же утверждение верно и для любого большого интервала скоростей, т.е. $v_{2}>$ $\delta>0$, где $\delta$ не зависит от $N$.

Аналогично термину "сходимость по мере", можно сказать, что в формуле (15) есть “оценка по мере". На самом деле, физическую формулу (1) мы можем переписать в более точном виде

$$
\mathscr{N}_{\Delta v}=\int_{v_{1}}^{v_{1}+O\left(N^{-1 / 2+\delta}\right)}\left(\frac{m}{2 \pi k T}\right)^{3 / 2} e^{-\left(m\left(v_{x}^{2}+v_{y}^{2}+v_{z}^{2}\right)\right) / 2 k T} d v_{x} d v_{y} d v_{z}
$$

где $\Delta=O\left(N^{-1 / 2+\delta}\right), \delta>0$.

В обычных в теории вероятностей обозначениях теорема звучит так.

Теорема 2. Имеет место соотношение

$$
\begin{aligned}
& \mathrm{P}\left(\mathscr{N}\left(\left|v_{1}\right|,\left|v_{1}\right|+O\left(N^{(-1 / 2)+\delta}\right)\right)\right. \\
& \left.\quad-\frac{\int_{\left|v_{1}\right|}^{\left|v_{1}\right|+O\left(N^{(-1 / 2)+\delta}\right)} e^{-m v^{2} / 2 k T} d v_{1} d v_{2} d v_{3}}{\int_{-\infty}^{-\infty} e^{-m v^{2} / 2 k T} d v_{1} d v_{2} d v_{3}} \geqslant \frac{\sqrt{\ln N}}{\sqrt{N}}|\ln \ln N|^{\varepsilon}\right) \\
& =O\left(N^{-k}\right),
\end{aligned}
$$

где $k$ - любое число, $\varepsilon>0$ - сколь угодно малое число, $\delta$ - любое число, $\left|v_{1}\right| \geqslant 0$ любые скорости. Здесъ Р есть лебегова мера фазового обгема, определенного в скобках (17), относительно всего обгема.

Эти оценки неулучшаемы. Теорема относится к теории чисел. Она не имеет отношения к динамике частиц, когда распределение Максвелла следует из уравнения Больцмана, которое до сих пор строго не обосновано. Обычный динамический подход и его критика содержится в работе Козлова [2]. 
Хотя конечно, естественно, что при определенных условиях динамиченская система приходит к наиболее вероятному с точки зрения вероятностной теории чисел распределению. Это соображение может быть полезно для динамического подхода.

Мы приведем достаточно элементарное доказательство теоремы о распределении Максвелла, по существу не ссылаясь на важные и красивые результаты теории чисел, опирающиеся на теорему Мейнардуса [3; теорема 6.2] и на красивую теорию мультипликативных мер Вершика [4], которая позволяет избежать некоторых непринципиальных и нарочитых введений целочисленности (например, взятия целой части от $\left.l^{1 / 2}\right)$.

Зато приведенные оценки, на которые автор опирался в работах по экономике и лингвистике [5], [6], являются доступными для менее искушенных в теории чисел и теории вероятности читателей, в частности, для физиков и специалистов по матанализу.

Доказательство основано на тех оценках, которые были приведены автором в [7], и теореме, аналогичной теореме Мейнардуса.

Для 6-мерного случая подробное доказательство дано в работе [8]. Мы по существу повторим это доказательство для 3-мерного случая.

Будем изучать систему, определяемую следующим образом. Для уровней энергии $j=0,1,2, \ldots$ кратностей

$$
q_{i}=\left[j^{1 / 2}\right], \quad j=0,1,2, \ldots,
$$

рассматриваются всевозможные наборы $\left\{N_{j k}\right\}$ неотрицательных целых чисел $N_{j k}$, $j=0,1,2, \ldots, k=1, \ldots, q_{i}$, удовлетворяющих условиям

$$
\begin{gathered}
\sum_{j=0}^{\infty} N_{j k}=N \\
\sum_{j=0}^{\infty} \sum_{k=1}^{q_{i}} j N_{j k} \equiv \sum_{j=1}^{\infty} \sum_{k=1}^{q_{i}} j N_{j k} \leqslant M,
\end{gathered}
$$

где $N$ и $M$ - заданные положительные числа (которые без ограничения общности будем считать целыми). Все такие наборы будем считать равновероятными.

Обозначим безразмерную величину $M=E / U_{0}$ и определим числа $\beta$ и $\xi$ как решения системы уравнений

$$
M=\xi^{-1} \sum_{j=1}^{\infty} j\left[j^{1 / 2}\right] e^{-\beta j}, \quad N=\xi^{-1} \sum_{j=0}^{\infty}\left[j^{1 / 2}\right] e^{-\beta j}
$$

ЗАмЕчАниЕ. В силу формулы Эйлера-Маклорена

$$
\begin{aligned}
& N=\xi^{-1} \sum_{0}^{\infty} x^{1 / 2} e^{-\beta x} d x(1+O(\beta))=\frac{1}{2} \sqrt{\pi} \beta^{-3 / 2} \xi^{-1}, \\
& M=\xi^{-1} \sum_{0}^{\infty} x^{3 / 2} e^{-\beta x} d x(1+O(\beta))=\frac{3}{4} \sqrt{\pi} \beta^{-5 / 2} \xi^{-1} .
\end{aligned}
$$

Отсюда $\beta$ и $\xi$

$$
\beta \cong \frac{2}{3} \frac{N}{M}, \quad \xi \cong \frac{1}{2} \sqrt{\pi} \frac{M^{3 / 2}}{N^{5 / 3}}
$$


Обозначим через $\mathscr{N}(M, N)$ общее число наборов $\left\{N_{j k}\right\}$, удовлетворяющих ограничениям (19), (20).

Всюду будет предполагаться, что параметры $\beta$ и $\xi$ удовлетворяют соотношению

$$
\xi<\beta^{-3 / 2+\varepsilon}
$$

для некоторого произвольного (но фиксированного) $\varepsilon>0$ ).

Положим

$$
\mu=\ln \xi, \quad \text { так что } \quad \xi=e^{\mu} .
$$

Пусть $\mathscr{M}$ - множество упорядоченных выборок, удовлетворяющих условиям (19) и $(20)$.

Для чисел $\mathscr{N}(N, M)$ таких вариантов мы получим следующую оценку:

$$
\mathscr{N}(M, N) \leqslant C \sqrt{N} \exp \{N \ln N+\beta M+\mu N\} .
$$

Действительно,

$$
\begin{aligned}
\mathscr{N}(M, N) & =N ! \sum_{\left\{N_{j k}\right\} \in \mathscr{M}} \frac{1}{\prod_{j=0}^{\infty} \prod_{k=1}^{q_{j}} N_{j k} !} \\
& \leqslant N ! e^{\beta M+\mu N} \sum_{\left\{N_{j k}\right\}} \frac{\exp \left\{-\sum_{j=0}^{\infty} \sum_{k=1}^{q_{j}} N_{j k}(\beta j+\mu)\right\}}{\prod_{j=0}^{\infty} \prod_{k=1}^{q_{j}} N_{j k} !} \\
& =N ! e^{\beta M+\mu N} \prod_{j=0}^{\infty} \prod_{k=1}^{q_{j}} \sum_{N_{j k}=0}^{\infty} \frac{e^{-N_{j k}(\beta j+\mu)}}{N_{j k} !} \\
& =N ! e^{\beta M+\mu N} \prod_{j=0}^{\infty} \prod_{k=1}^{q_{j}} e^{e^{-\beta j-\mu}} \\
& =N ! \exp \left\{\beta M+\mu N+\sum_{j=0}^{\infty} q_{j} e^{-\beta j-\mu}\right\} \\
& =N ! \exp \{\beta M+\mu N+N\} \\
& \leqslant C \sqrt{N} \exp \{N \ln N+\beta M+\mu N\}
\end{aligned}
$$

Пусть $\mathscr{M}_{\Delta} \subset \mathscr{M}-$ подмножество вариантов таких, что

$$
\left|\sum_{j=0}^{l} \sum_{k=1}^{q_{j}}\left(N_{j k}-\bar{N}_{j k}\right)\right|>\Delta
$$

где

$$
\bar{N}_{j k}=e^{-\beta j-\mu} \equiv \frac{\bar{N}_{j}}{q_{j}} .
$$

Для числа $\mathscr{N}(M, N, \Delta)$ выборки из $\mathscr{M}_{\Delta}$ мы получим оценку

$$
\mathscr{N}(M, N, \Delta) \leqslant N ! \exp \left\{\beta M+\mu N-c \Delta+\sum_{j=l+1}^{\infty} q_{j} e^{-\beta j-\mu}\right\}
$$




$$
\times\left(\exp \left\{\sum_{j=0}^{l}\left(q_{j} e^{-\beta j-\mu+c}-c \bar{N}_{j}\right)\right\}+\exp \left\{\sum_{j=0}^{l}\left(q_{j} e^{-\beta j-\mu-c}+c \bar{N}_{j}\right)\right\}\right)
$$

для $0<c<\mu$, где $\bar{N}_{j}$ задается формулой $(24)$.

Далее возьмем два члена разложения по формуле Тейлора, как в [9],

$$
q_{j} e^{-\beta j-\mu \pm c} \mp c \bar{N}_{j}=q_{j} e^{-\beta j-\mu}\left(e^{ \pm c} \mp c\right)=q_{j} e^{-\beta j-\mu}\left(1+\frac{c^{2}}{2} e^{ \pm \theta c}\right),
$$

где $\theta(i)$ - некоторая средняя точка, $\theta \equiv \theta(c) \in(0,1)$.

Если $c \leqslant \min \{\mu / 2,1\}$, то отсюда следует неравенство

$$
\sum_{j=0}^{l} q_{j} e^{\beta j+\mu-\theta_{j} c} \leqslant 2 K e^{-\mu} \beta^{-3 / 2}
$$

где $K$ - константа.

Следовательно,

$$
\mathscr{N}(N, M, \Delta) \leqslant C \sqrt{N} \exp \{N \ln N+\beta M+\mu N\} \exp \left\{-c \Delta+K c^{2} e^{-\mu} \beta^{-3 / 2}\right\} .
$$

Мы подставим

$$
\Delta=\sqrt{N \ln N}|\ln \ln N|^{\varepsilon} \asymp e^{-\mu / 2} \beta^{-3 / 2} \sqrt{\ln N}|\ln \ln N|^{\varepsilon}
$$

и

$$
c=\frac{\beta^{3 / 2} e^{\mu} \Delta}{2 K} \asymp \beta^{3 / 4} e^{\mu / 2} \sqrt{\ln N}|\ln \ln N|^{\varepsilon}
$$

в (28). Отсюда следует, в частности,

$$
\mathscr{N}(N, M, \Delta) \leqslant C_{k} \sqrt{N} \exp \{N \ln N+\beta M+\mu N\} N^{-k}
$$

для любого $k$.

Теперь оценим снизу эти же величины.

Мы оценим число выборок $\mathscr{N}_{0}(M, N)<\mathscr{N}(M, N)$, удовлетворяющих условиям (19) и (20), причем в последнем неравенстве положим равенство

$$
\sum_{j=0}^{\infty} \sum_{k=1}^{q_{j}} N_{j k}=N, \quad \sum_{j=0}^{\infty} \sum_{k=1}^{q_{j}} j N_{j k}=M
$$

Пусть $\mathscr{M}_{0}-$ множество наборов чисел заполнения, удовлетворяющих (32). Тогда

$$
\begin{aligned}
\mathscr{N}_{0}(M, N) & =\sum_{\left\{N_{j k}\right\} \in \mathscr{M}_{0}} \frac{N !}{\prod_{j=0}^{\infty} \prod_{k=1}^{q_{j}} N_{j k} !} \\
& =N ! \sum_{\left\{N_{j k}\right\}} \frac{\delta\left(N, \sum_{j=0}^{\infty} \sum_{k=1}^{q_{j}} N_{j k}\right) \delta\left(M, \sum_{j=0}^{\infty} \sum_{k=1}^{q_{j}} j N_{j k}\right)}{\prod_{j=0}^{\infty} \prod_{k=1}^{q_{j}} N_{j k} !} .
\end{aligned}
$$


Здесь сумма во второй строке берется по всем финитным наборам неотрицательных чисел заполнения, а

$$
\delta(m, n) \equiv \delta_{m n}
$$

- символ Кронекера.

Подставим интегральное представление

$$
\delta_{m n}=\frac{s}{2 \pi} \int_{-\pi / s}^{\pi / s} e^{(i s x+\omega)(m-n)} d x
$$

символа Кронекера (где $s$ и $\omega$ - произвольные ненулевые вещественные числа) в (33), выбирая $s=1$ и $\omega=\mu$ для первого сомножителя и $s=\omega=\beta$ для второго сомножителя. Тогда для $\mathscr{N}_{0}(M, N)$ мы получим интегральное представление

$$
\mathscr{N}_{0}(M, N)=\frac{\beta N ! e^{\beta M+\mu N}}{4 \pi^{2}} \int_{-\pi / \beta}^{\pi / \beta}\left(\int_{-\pi}^{\pi} e^{\Lambda \Phi(\varphi, \psi)} d \psi\right) d \varphi
$$

где

$$
\begin{gathered}
\Lambda=\beta^{-3 / 2} e^{-\mu} \asymp N \\
\Phi(\varphi, \psi)=i \beta^{5 / 2} e^{\mu} M \varphi+i \beta^{3 / 2} e^{\mu} N \psi+\beta^{3 / 2} \sum_{j=0}^{\infty} q_{j} e^{-\beta j-i(\psi+\beta j \varphi)} .
\end{gathered}
$$

(Знак $\asymp N$ означает, что существуют константы $c_{1}$ и $c_{2}$, такие, что $c_{1} N \leqslant \lambda \leqslant c_{2} N$ ). Действительно, указанная выше подстановка дает

$$
\begin{aligned}
\mathscr{N}_{0}(M, N) & =\frac{\beta N ! e^{\beta M+\mu N}}{4 \pi^{2}} \\
& \times \int_{-\pi / \beta}^{\pi / \beta}\left(\int_{-\pi}^{\pi} \sum_{\left\{N_{j k}\right\}} \frac{e^{i \beta \varphi M+i \psi N}}{\prod_{j=0}^{\infty} \prod_{k=1}^{q_{j}} N_{j k} !}\right. \\
& \left.\times \exp \left\{-\sum_{j=0}^{\infty} \sum_{k=1}^{q_{j}} N_{j k}(\beta j+\mu+i \beta j \varphi+i \psi)\right\} d \psi\right) d \varphi \\
= & \frac{\beta N ! e^{\beta M+\mu N}}{4 \pi^{2}} \int_{-\pi / \beta}^{\pi / \beta}\left(\int_{-\pi}^{\pi} e^{i \beta \varphi M+i \psi N} \prod_{j=0}^{\infty} \prod_{k=1}^{q_{j}} \sum_{N_{j k}=0}^{\infty} \frac{e^{-N_{j k}(\beta j+\mu+i \beta j \varphi+i \psi)}}{N_{j k} !} d \psi\right) d \varphi \\
= & \frac{\beta N ! e^{\beta M+\mu N}}{4 \pi^{2}} \int_{-\pi / \beta}^{\pi / \beta}\left(\int_{-\pi}^{\pi} e^{i \beta \varphi M+i \psi N} \prod_{j=0}^{\infty} \prod_{k=1}^{q_{j}} \exp \left\{e^{-(\beta j+\mu+i \beta j \varphi+i \psi)}\right\} d \psi\right) d \varphi \\
= & \frac{\beta N ! e^{\beta M+\mu N}}{4 \pi^{2}} \int_{-\pi / \beta}^{\pi / \beta}\left(\int_{-\pi}^{\pi} \exp \left\{i \beta \varphi M+i \psi N \sum_{j=0}^{\infty} q_{j} e^{-(\beta j+\mu+i \beta j \varphi+i \psi)}\right\} d \psi\right) d \varphi .
\end{aligned}
$$

ЛЕмма 1. Фазовая функиия $\Phi(\varphi, \psi)$, задаваемая формулой (36), обладает следующими свойствами:

1) все ее производные равномерно ограничены при значениях $\beta$ и $\xi$, удовлетворяющих неравенству (21); 
2) фазовая функиия имеет стационарную точку $\varphi=0 \bmod 2 \pi / b, \psi=0 \bmod 2 \pi$;

3) матрица $\Phi^{\prime \prime}(0,0)$ вторьх производных фазовой функции в стационарной точке невырождена и равномерно по параметрам $\beta$ и $\xi$, удовлетворяющим неравенству (21), строго отрицательно определена;

4) мнимая часть фазовой функции в стационарной точке равна нулю, а ее вещественная часть достигает там абсолютного максимума, причем для любого $\gamma>0$ существует такое $\delta>0$, не зависящее от параметров $\beta$ и удовлетворяющих неравенству (21), что

$$
\operatorname{Re} \Phi(\varphi, \psi)<\operatorname{Re} \Phi(0,0)-\delta \quad \partial л я \quad \operatorname{dist}((\varphi, \psi),(0,0))>\gamma
$$

ДокАЗАТЕЛЬСтво. 1) Ограниченность производных фазовой функции доказывается непосредственной выкладкой.

2) Чтобы проверить, что точка $(0,0)$ есть стационарная точка фазовой функции, вычислим ее первые производные:

$$
\begin{aligned}
& \frac{\partial \Phi}{\partial \varphi}=i \beta^{5 / 2} e^{\mu}\left[M-e^{-\mu} \sum_{j=0}^{\infty} j q_{j} e^{-\beta j-i(\psi+\beta j \varphi)}\right], \\
& \frac{\partial \Phi}{\partial \psi}=i \beta^{3 / 2} e^{\mu}\left[N-e^{-\mu} \sum_{j=0}^{\infty} q_{j} e^{-\beta j-i(\psi+\beta j \varphi)}\right] .
\end{aligned}
$$

При $\varphi=\psi=0$ обе производные обращаются в нуль в силу определения параметров $\beta$ и $\xi=e^{\mu}$.

3) Матрица $\Phi^{\prime \prime}(0,0)$ имеет вид

$$
\Phi^{\prime \prime}(0,0)=-\sum_{j=0}^{\infty} \beta^{3 / 2} q_{j} e^{-\beta j}\left(\begin{array}{cc}
1 & \beta j \\
\beta j & \beta^{2} j^{2}
\end{array}\right) .
$$

Оценим эту матрицу как матрицу квадратичной формы следующим образом:

$$
\Phi^{\prime \prime}(0,0) \leqslant-\sum_{j=\left[x_{1} / \beta\right]}^{\left[x_{2} / \beta\right]} \beta^{3 / 2} q_{j} e^{-\beta j}\left(\begin{array}{cc}
1 & \beta j \\
\beta j & \beta^{2} j^{2}
\end{array}\right),
$$

где $x_{2}>x_{1}>0$ - произвольные фиксированные числа.

При малых $\beta$ матрицу в правой части с учетом асимптотики $q_{j} \simeq j / 2$ при больших $j$ можно вычислить по формуле Эйлера-Маклорена, получая в результате с точностью до $о(1)$ матрицу

$$
-\frac{1}{2}\left(\begin{array}{cc}
\int_{x_{1}}^{x_{2}} x e^{-x} d x & \int_{x_{1}}^{x_{2}} x^{3 / 2} e^{-x} d x \\
\int_{x_{1}}^{x_{2}} x^{3 / 2} e^{-x} d x & \int_{x_{1}}^{x_{2}} x^{2} e^{-x} d x
\end{array}\right)=-\frac{1}{2}\left(\begin{array}{ll}
(1,1) & (1, x) \\
(x, 1) & (x, x)
\end{array}\right),
$$

где

$$
(u, v)=\int_{x_{1}}^{x_{2}} u(x) \bar{v}(x) e^{-x} x d x
$$

- скалярное произведение $L^{2}\left(\left[x_{1}, x_{2}\right], e^{-x} x\right)$. 
Поскольку функции 1 и $x$ линейно независимы, матрица (43) отрицательно определена, что и доказывает требуемое утверждение.

4) Из формулы $(36)$ для $\Phi(\varphi, \psi)$ получаем

$$
\operatorname{Re} \Phi(0,0)-\operatorname{Re} \Phi(\varphi, \psi)=\beta^{3 / 2} \sum_{j=0}^{\infty} q_{j} e^{-\beta j}(1-\cos (\psi+\beta j \varphi))
$$

Все слагаемые в правой части здесь неотрицательны. Поэтому, опуская часть из них и оценивая коэффициенты $\beta q_{j} e^{-\beta j}$ для оставшихся слагаемых, получаем

$$
\begin{aligned}
\operatorname{Re} \Phi(0,0)-\operatorname{Re} \Phi(\varphi, \psi) & \geqslant \operatorname{const} \beta \sum_{j=\left[x_{1} / \beta\right]}^{\left[x_{2} / \beta\right]}(1-\cos (j \varphi+\psi)) \\
& \geqslant \operatorname{const}\left(x_{2}-x_{1}-\beta-\beta\left|\sin \frac{\beta \varphi}{2}\right|^{-1}\right) .
\end{aligned}
$$

Подбирая $x_{1}$ и $x_{2}$ подходящим образом, получаем требуемое утверждение. Лемма доказана.

Доказанная лемма позволяет вычислить интеграл (34) по методу перевала и получить оценку снизу для числа упорядоченных выборок в виде

$$
\mathscr{N}(N, M) \geqslant C \beta \Lambda^{-1} \sqrt{N} \exp \{N \ln N+\beta M+\mu N\} .
$$

Теперь мы можем оценить интеграл (37). По лемме 1 все производные функции $\Phi(\varphi)$ равномерно ограничены. Кроме того, если представить ее в виде $\Phi=$ $\Phi_{1}+i \Phi_{2}$, где $\Phi_{1}$ и $\Phi_{2}$ вещественны, то,

$$
\Phi_{1}^{\prime}(0)=0, \quad \Phi_{1}^{\prime \prime}(0)<-C<0, \quad \Phi_{2}^{\prime}(0)=\Phi_{2}^{\prime \prime}(0)=0 .
$$

Отсюда с помощью формулы Тейлора с остаточным членом получаем при $|\varphi| \leqslant \varepsilon$, где $\varepsilon>0$ достаточно мало, оценки

$$
\begin{gathered}
\Phi(0)-C_{1}|\varphi|^{2} \leqslant \Phi_{1}(\varphi) \leqslant \Phi(0)-C_{2}|\varphi|^{2}, \\
\left|\Phi_{2}(\varphi)\right| \leqslant C_{3}|\varphi|^{3}
\end{gathered}
$$

с положительными постоянными $C_{j}$, не зависящими от $M$ и последовательности $\left\{R_{j}\right\}$.

Пусть

$$
1=\psi_{1}(\varphi)+\psi_{2}(\varphi)
$$

- неотрицательное гладкое разбиение единицы на окружности $S^{1} \ni \varphi$ радиуса $b$ такое, что

$$
\operatorname{supp} \psi_{1} \subset[-\varepsilon, \varepsilon] \quad \text { и } \quad \psi_{1}(\varphi)=1 \quad \text { при } \varphi \in\left[-\frac{\varepsilon}{2}, \frac{\varepsilon}{2}\right] .
$$

Представим интеграл

$$
I=\int_{S^{1}} \exp \left\{\beta^{-3 / 2} \Phi(\varphi)\right\} d \varphi
$$


в виде суммы

$$
I=\int_{S^{1}} \exp \left\{\beta^{-3 / 2} \Phi(\varphi)\right\} \psi_{1}(\varphi) d \varphi+\int_{S^{1}} \exp \left\{\beta^{-3 / 2} \Phi(\varphi)\right\} \psi_{2}(\varphi) d \varphi \equiv I_{1}+I_{2}
$$

По лемме 1,3 )

$$
\operatorname{Re} \Phi(\varphi) \leqslant \Phi(0)-\delta, \quad \delta>0
$$

на носителе подынтегрального выражения в $I_{2}$, а мера носителя имеет порядок $\beta^{-1}$. Поэтому

$$
\left|I_{2}\right| \leqslant K \exp \left\{\frac{\beta^{-3 / 2} \delta}{2}+\beta^{-3 / 2} \Phi(0)\right\}, \quad b \rightarrow 0,
$$

с некоторой постоянной $K$.

Оценим теперь интеграл $I_{1}$. Для удобства обозначим временно через $h=\beta^{3 / 2}$ малый параметр, который стоит в экспоненте нашего интеграла. В отрезке $D=$ $[-\varepsilon, \varepsilon]$ выделим два подотрезка $D_{1 / 2} \subset D_{1 / 3} \subset D$, полагая

$$
D_{1 / 2}=\left[-\varepsilon h^{1 / 2}, \varepsilon h^{1 / 2}\right], \quad D_{1 / 3}=\left[-\varepsilon h^{1 / 3}, \varepsilon h^{1 / 3}\right] .
$$

Тогда

$$
\left|\frac{\Phi_{2}}{h}\right| \leqslant C_{3} \varepsilon^{3} \quad \text { при } \quad \varphi \in D_{1 / 3},
$$

так что (при достаточно малом $\varepsilon$ ) мнимая часть аргумента экспоненты $D_{1 / 3}$ мала и справедливо соотношение

$$
\operatorname{Re} e^{\Phi(\varphi) / h} \geqslant \frac{1}{2} e^{\Phi_{1}(\varphi) / h}, \quad \varphi \in D_{1 / 3} .
$$

Далее,

$$
\frac{\Phi(0)}{h} \geqslant \frac{\Phi_{1}(\varphi)}{h} \geqslant \frac{\Phi(0)}{h}-C_{1} \varepsilon^{2}, \quad \varphi \in D_{1 / 2} .
$$

Комбинируя это с предыдущим неравенством и учитывая, что длина интервала $D_{1 / 2}$ равна $2 \varepsilon h^{1 / 2}$, получаем

$$
\operatorname{Re} \int_{D_{1 / 2}} e^{\Phi(\varphi) / h} \psi_{1}(\varphi) d \varphi \geqslant C_{4} e^{\Phi(0) / h} h^{1 / 2} .
$$

Далее,

$$
\operatorname{Re} \int_{D_{1 / 3} \backslash D_{1 / 2}} e^{\Phi(\varphi) / h} \psi_{1}(\varphi) d \varphi \geqslant 0
$$

в силу (50). Кроме этого, справедливо неравенство

$$
\frac{\Phi_{1}(\varphi)}{h} \leqslant \frac{\Phi_{1}(0)}{h}-C_{2} \varepsilon^{2} h^{-1 / 3}, \quad \varphi \in D \backslash D_{1 / 3},
$$

так что

$$
\left|\int_{D \backslash D_{1 / 3}} e^{\Phi(\varphi) / h} \psi_{1}(\varphi) d \varphi\right| \leqslant C_{5} e^{\Phi(0) / h} e^{-C_{2} \varepsilon^{2} h^{-1 / 3}} .
$$

Комбинируя все предыдущие оценки, получаем

$$
I=\operatorname{Re} I \geqslant C_{6} \beta^{3 / 4} \exp \left\{\beta^{-3 / 2} \Phi(0)\right\} .
$$


Остается подставить эту оценку в формулу (37) для $\mathscr{N}_{0}(M, N)$ с учетом формулы (36) для фазовой функции, и в силу неравенства $\mathscr{N}(M, N)>\mathscr{N}_{0}(M, N)$ мы приходим к оценке снизу для $\mathscr{N}(M, N)$. В результате получаем теорему 1. В силу того, что число $\mathscr{N}(M, N)$ отвечает лебеговой мере всего фазового объема, а число $\mathscr{N}(M, N, \Delta)$ отвечает лебеговой мере фазового объема, определенного в скобках в формуле (17), теорема 2 доказана.

Как уже отмечалось в [10], использование неупорядоченной выборки с возвращением приводит нас к математической формуле для бозе-газа, однако, без параметра $\hbar$ - константы Планка, а с теми же параметрами, которые у нас возникли при использовании параметров потенциала взаимодействия Леннарда-Джонса. Вместо формулы (16) мы получаем следующую формулу:

$$
\mathscr{N}_{\Delta v}=\frac{1}{2.612} \int_{v_{1}}^{v_{1}+\Delta v}\left(\frac{m}{2 \pi k T}\right)^{3 / 2} \frac{1}{e^{m v^{2} / 2 k T}-1} d v_{x} d v_{y} d v_{z} .
$$

Однако $\Delta v$ на этот раз зависит от $v_{1}$. А именно, если $v_{1} \sim 1$, то $\Delta v=N_{0}^{-1 / 2+\delta}$, где $\delta>0$, а $N_{0}$ - число частиц, насыщающее объем $V$ при темпетатуре $T$ и заданной максимальной энергии $E / U_{0}$,

$$
N_{0}=\left(\frac{m}{U_{0}}\right)^{3 / 2} \int_{0}^{\infty} \frac{d v_{x} d v_{y} d v_{z}}{e^{\left(\kappa v^{2}\right) / 2 m T}-1} .
$$

По нашим исходным параметрам скорость может быть выражена в виде $v=$ $\sqrt{U_{0} / 2 m}$. Пусть $v_{0}$ - минимальная скорость - равна $v_{0}=v N^{-1 / 3+\delta}$, где $\delta>0$ определяет малость $v_{0}$. При $\delta=1 / 3$ получаем $v_{0}=v$, поэтому полагаем $1 / 3>\delta>0$. Оценка ошибки в формуле распределения (52) будет иметь вид

$$
O\left(N^{-1 / 3-\delta / 2} \sqrt{\ln N}(\ln \ln N)^{\varepsilon}\right),
$$

а именно, имеет место следующая теорема.

Теорема 3. Имеет место соотношение

$$
\begin{aligned}
& \mathrm{P}\left(\mathscr{N}_{\Delta v}-\frac{1}{2.612} \int_{\left|v_{0}\right|}^{\left|v_{0}\right|+O\left(N_{0}^{-1 / 3+\delta_{1}}\right)}\left(\frac{m}{2 \pi k T}\right)^{3 / 2} \frac{1}{e^{m v^{2} / 2 k T}-1} d v_{x} d v_{y} d v_{z}\right. \\
& \left.\geqslant N^{-1 / 3-\delta / 2} \sqrt{\ln N}(\ln \ln N)^{\varepsilon}\right) \\
& \leqslant O\left(N_{0}^{-k}\right),
\end{aligned}
$$

где $k$ - любое целое, $\varepsilon>0$ - сколь угодно малое, $v_{0} \geqslant 0,0<\delta<1 / 3, \delta_{1}>\delta$, $v=\sqrt{U_{0} / 2 m}, \Delta v=O\left(N_{0}^{-1 / 3+\delta_{1}}\right.$. Здесь $\mathrm{P}$ есть лебегова мера фазового обгема, определенного в скобках (54), относителъно всего обгема.

Физикам трудно осознать эту задачу, поскольку при этом возникает математический эффект типа бозе-конденсации, т.е. такого насыщения, после которого возникает "бозе-конденсат", который, с точки зрения автора, может быть протрактован, например, как некая коагуляция частиц низких скоростей и образование димеров, тримеров и других кластеров. 
Обычно явление появления димеров пытаются получить моделированием, которое начинается с начальных условий и привлечения взаимодействия, например, Леннарда-Джонса. Согласно концепции автора, если это взаимодействие, то такое, которое может осуществляться до включения взаимодействия типа Леннарда-Джонса: с точки зрения удельного объема мы пока еще находимся в ситуации идеального газа. Такого сорта взаимодействие наблюдается, например, для газа $C_{60}$ (фуллерена), обладающего очень слабым притяжением (порядка $O\left(1 / r^{9}\right)$ ). Оно связано с асимметрией молекул и с тем, какими сторонами соприкоснулись молекулы $C_{60}$.

Это наблюдать экспериментально значительно легче, поскольку фуллерен не имеет жидкой фазы, а сразу превращается в твердые частицы фуллерита.

Наличие такого насыщенного общего числа частиц в поставленной задаче, выше которого частица должна куда-то деться (перейти в бозе-конденсат), ${ }^{1}$ это математический строго доказанный факт, так же, как и четкие оценки того, где могут возникать скопления. Однако не совсем корректно сказано, что добавлены частицы. На самом деле лучше сказать, что мы понизили температуру, увеличивая поршнем давление, и, значит, насыщающее общее число частиц уменьшилось. А можно просто говорить, что при данной температуре давление увеличивается до тех пор, пока не возникает $\lambda$-переход в "бозе-конденсат". Спрашивается, куда делись остальные частицы, если понижение температуры произведено одновременно с ограничением давления или давление при данной температуре стало достаточно велико? Может быть, они осели на стенках сосуда? Такой закон "обязательного" оседания (коагуляции) на стенках был бы еще более интересен и имел бы важное практическое приложение. Однако эксперименты в большей степени подталкивают к первой точке зрения. У физиков даже существует мнение, что переход к димерам - это фазовый переход.

Самое существенное то, что эта оценка не улучшаема. Этот факт следует из теоремы 2 работы [11]. Он позволяет определить в случае насыщения, сколько частиц при понижении температуры и поддержании постоянного давления поршнем переходят в кластеры (см. [12]). Это решает и проблему парадокса Гиббса.

Феноменологическая термодинамика основывается на парном взаимодействии. При этом неявно предполагается, что существует некое "среднее поле", образованное $N$ частицами. Другими словами, предполагается, что существует некоторое одночастичное распределение, характеризующее его, или некоторый средний потенциал, зависящий от импульсов и координат, создаваемый всеми частицами. Они связаны между собой. Формулы для распределения, отвечающие этому среднему полю, строго получены автором в работе [13]. Уравнение, которое связывает потенциал среднего поля и парное взаимодействие, называется уравнением самосогласованного (или среднего) поля. Оно имеет вид для потенциала взаимодействия $\Phi$

$$
u(x, p)=u_{0}(x, p)+N \int \Phi\left(x-x^{\prime}, p-p^{\prime}\right) \frac{1}{e^{\left(p^{2} / 2 m+u\left(x^{\prime}, p^{\prime}\right)\right)(k T)^{-1}}-1} d x^{\prime} d p^{\prime} .
$$

Это уравнение строго обосновано лишь в случае дальнодействия, в частности, в работе [14].

\footnotetext{
${ }^{1}$ Физики, которым я рассказывал эту теорию, предупреждали меня, чтобы я не произносил слова "бозе-конденсат", поскольку это вызывает у них ассоциации, затрудняющие понимание предложенной концепции.
} 
В случае газа, занимающего объем $V$, на который не действуют никакие внешние силы, $u_{0}(x, p)=0$ при $x$, лежащих внутри объема $V$, и $u_{0}(x, p)=\infty$ на границе этого объема.

Этот случай мы и будем в дальнейшем изучать. В качестве потенциала взаимодействия мы примем потенциал Леннарда-Джонса или для более точного совпадения с экспериментом потенциал вида

$$
\Phi(r)=\frac{\varepsilon n}{n-6}\left(\frac{n}{6}\right)^{6 /(n-6)}\left(\frac{\sigma^{n}}{r^{n}}-\frac{\sigma^{6}}{r^{6}}\right),
$$

содержащий еще один параметр $n>6$.

В работе [13] мы уже модифицировали это выражение, используя задачу рассеяния для потенциала Леннарда-Джонса, сводя его к интегрированию по углу рассеяния. В настоящей работе мы не будем использовать угол рассеяния, а только прицельный параметр, и исправив опечатки, избавимся от малого параметра $\kappa$, как это и делают физики в распределении Максвелла, так, чтобы можно было просчитывать окончательные формулы непосредственно с использованием вычислительной техники.

Сначала коснемся истории возникновения редукции потенциала взаимодействия с помощью задачи рассеяния. Впервые, насколько мне известно, такая мысль возникла у Л. Д. Ландау на докладе Н. Н. Боголюбова о сверхтекучести слабо-неидеального бозе-газа в 1947 г.

Дело в том, что Боголюбов, используя технику вторичного квантования, доказал достаточно строго для случая условий Борна-Кармана, т.е. на торе, причем далее перешел к пределу при радиусе тора, стремящемся к бесконечности (этот последний переход некорректен, но более понятен физикам), для слабо неидеального бозе-газа условие сверхтекучести в случае отталкивающего потенциала. В формулу, полученную Боголюбовым, вошло преобразование Фурье от этого потенциала, которое, разумеется, существенно зависит от $r_{0}$ - точки обрезания потенциала Леннарда-Джонса.

Как пишет Боголюбов, Ландау заметил, что “так как при малой плотности взаимодействия между молекулами осуществляется главным образом с помощью именно парных соударений, то выражение (30) должно быть заменено соответствующим выражением, пропорциональным амплитуде "точной вероятности" парного соударения" [15; с. 222-223] Эту программу затем осуществил С. Т. Беляев с помощью диаграмной техники Фейнмана. Автор обобщил технику вторичного квантования (назвав новую технику ультравторичным квантованием), чтобы получить сверхтекучесть классических газов в капилляре и в нанотрубке (см. [16]-[18]), что получило экспериментальное подтверждение [19]-[22].

Поскольку обнаружились ранее не известные для меня и круга моих знакомых физиков экспериментальные данные, то позволю себе более подробно остановиться на этом вопросе.

Теоретические вопросы о сверхтекучести в нанотрубках я получил как математик, а математик, по определению одного из известных английских физиков, это “человек свободный от законов природы". Это значит, что я вскрыл математическую подоплеку работ Боголюбова и Ландау о сверхтекучести и получил из их предпосылок парадоксальный результат о том, что классический газ (не квантовый) может "сверхтечь", и что на самом деле сверхтекучесть не есть свойство квантовой жидкости, а свойство протекания по достаточно узкому капилляру. И для капилляра 
достаточно малого радиуса (нанотрубки) классический газ с четным числом нейтронов может быть сверхтекуч. Это значит, что у него существует метастабильное состояние с достаточно высокими барьерами (высота их зависит от числа частиц), которые обеспечивают достаточно длительное квазистационарное состояние с постоянной скоростью.

Этот результат мог означать, что исходные идеи Боголюбова и Ландау (их "аксиоматика") неполны. Но последние эксперименты, показывающие превышение минимум на 1-2 порядка теории Кнудсона, возможно, говорят о другой, неквантовой, природе сверхтекучести и, возможно, подтверждают мои парадоксальные выводы. Как было мной показано [23], формулы Боголюбова выдерживают переход при $h \rightarrow 0$, если рассматривать задачу во внешнем поле (как обычно, без внешнего поля квазиклассика совпадает с точным решением квантовой задачи, и малость параметра $h$ как признак медленно меняющегося внешнего поля не чувствуется). Действительно, кривая Ландау содержит фононы и ротоны. Первые, естественно, сохраняются при переходе в классику, а вторые при потенциале взаимодействия, равном нулю, превращаются в идельный газ с энергией $p^{2} / 2 m$, где $p$ - импульс, $m-$ масса. Что касается спина, то Фейнман задолго до работы автора [24], заметил, что спин сохраняется в классической теории, как и поляризация в геометрической оптике. Четность или нечетность числа нейтронов определяют спин и статистики (см. статьи автора о классических фермионах и бозонах [25], [26], [27]).

Тем не менее, удобнее рассматривать квантовые уравнения без внешнего поля. Метод ультравторичного квантования приводит к следующим уравнениям в капилляре. Для нечетного числа нейтронов общее уравнение (33) работы [28] нужно брать при $\alpha=1 / 2$. Это приведет к следующему оператору:

$$
\begin{aligned}
\Omega \Psi(x, y)=- & \frac{\hbar^{2}}{2 m}\left(\Delta_{x}+\Delta_{y}\right) \Psi(x, y) \\
& +\iint d \xi d \eta(\Phi(x, \xi)+\Phi(y, \eta))\left(\Psi^{+}(\xi, \eta) \Psi(\xi, \eta) \Psi(x, y)\right. \\
\Omega \Psi^{+}(x, y)=- & \frac{\hbar^{2}}{2 m}\left(\Delta_{x}+\Delta_{y}\right) \Psi^{+}(x, y) \\
& +\iint d \xi d \eta(\Phi(x, \xi)+\Phi(y, \eta))\left(\Psi(\xi, \eta) \Psi^{+}(\xi, \eta) \Psi^{+}(x, y)\right.
\end{aligned}
$$

и далее к системе уравненияй в вариациях следующего вида:

$$
\begin{aligned}
(\Omega- & \lambda) F(x, y) \\
= & -\frac{\hbar^{2}}{2 m}\left(\Delta_{x}+\Delta_{y}\right) F(x, y) \\
& +\iint d \xi d \eta(\Phi(x, \xi)+\Phi(y, \eta))\left(G(\xi, \eta) \Psi(\xi, \eta) \Psi(x, y)+\Psi^{+}(\xi, \eta) F(\xi, \eta) \Psi(x, y)\right. \\
& \left.+\Psi^{+}(\xi, \eta) \Psi(\xi, \eta) F(x, y)\right) \\
& -\iint d \xi d \eta(\Phi(x, \xi)+\Phi(y, \eta))\left(G(\xi, \eta) \Psi(x, \eta) \Psi(\xi, y)+\Psi^{+}(\xi, \eta) F(x, \eta) \Psi(\xi, y)\right. \\
& \left.\quad+\Psi^{+}(\xi, \eta) \Psi(x, \eta) F(\xi, y)\right),
\end{aligned}
$$




$$
\begin{aligned}
(\Omega+ & \lambda) G(x, y) \\
= & -\frac{\hbar^{2}}{2 m}\left(\Delta_{x}+\Delta_{y}\right) G(x, y) \\
& +\iint d \xi d \eta(\Phi(x, \xi)+\Phi(y, \eta))\left(F(\xi, \eta) \Psi^{+}(\xi, \eta) \Psi^{+}(x, y)+\Psi(\xi, \eta) G(\xi, \eta) \Psi^{+}(x, y)\right. \\
& \left.+\Psi(\xi, \eta) \Psi^{+}(\xi, \eta) G(x, y)\right) \\
& -\iint d \xi d \eta(\Phi(x, \xi)+\Phi(y, \eta))\left(F(\xi, \eta) \Psi^{+}(x, \eta) \Psi^{+}(\xi, y)+\Psi(\xi, \eta) G(x, \eta) \Psi^{+}(\xi, y)\right. \\
& \left.+\Psi(\xi, \eta) \Psi^{+}(x, \eta) G(\xi, y)\right) .
\end{aligned}
$$

Для потенциала взаимодействия удобно использовать модель Сузерленда

$$
\Phi(x-y)=-\frac{\sigma^{4}}{|x-y|^{4}}
$$

при $r=|x-y| \geqslant \delta$ и при $r<\delta$ равен бесконечности. возникает кривая Ландау, но спектр существенно отличается от боголюбовского. Он имеет вид

$$
\frac{k^{2}}{2 m}+\widetilde{\Phi}(k)-\widetilde{\Phi}(0) .
$$

Фотонная часть зависит от коэффициента при (61), который, как известно, очень мал.

Наиболее интересные результаты, однако, должны получиться при нахождении решений вида вихрей, как для уравнений Гросса-Питаевского [29]. Такие решения (59) для взаимодействия Сузерленда легко находятся.

Теперь рассмотрим диаграмму Хоугена-Ватсона, приведенную в учебнике Бруштейна "Молекулярная физика" [30]. Диаграмма отражает зависимость фактора сжимаемости $Z=P V / k T N$ от давления для разных температур и была построена Хоугеном и Ватсоном в свое время для семи газов: $\mathrm{H}_{2}, \mathrm{~N}_{2}, \mathrm{CO}, \mathrm{NH}_{3}, \mathrm{CH}_{4}, \mathrm{C}_{3} \mathrm{H}_{8}$, $\mathrm{C}_{5} \mathrm{H}_{12}$. Хотя в учебнике говорится, что притяжение уменьшает сжимаемость, однако получается это для газа Ван дер Ваальса при условии $|1-Z| \ll 1$, т.е. время как фактор сжимаемости уменьшается до значения 0.2 .

Из нашей концепции идеального газа из распределения (52) получается, что $Z$ может достичь значения 0.523, а далее фактор сжимаемости должен уменьшаться уже за счет взаимодействия. Кроме того, из диаграммы видно, так же как из диаграммы, приведенной в предыдущей работе [13; рис.4], что существует точка, в которой $d Z / d V_{\text {уд }}=-\infty$. Нашей задачей, в частности, будет, учитывая взаимодействие Леннарда-Джонса, найти эту точку. Мы будем называть ее $\lambda$-точкой по аналогии с $\lambda$-точкой гелия- 4 .

Остановимся на определении уровня энергии, ниже которого начинается "конденсат".

Как известно, “точка поворота" $r_{0}$, энергия $E=m\left(v_{1}-v_{2}\right)^{2}$, где $v_{1}$ и $v_{2}-$ скорости двух взаимодействующих частиц, и прицельный параметр $\rho$ связаны соотношением

$$
E-\frac{E \rho^{2}}{r_{0}^{2}}-\Phi\left(r_{0}\right)=0 .
$$


1. Потенциал имеет вид $-\alpha / r^{4}$. Тогда точка поворота $r_{0}$ в задаче рассеяния определяется из соотношения

$$
1-\frac{\rho^{2}}{r_{0}^{2}}+\frac{\alpha}{r^{4} E}=0
$$

где $E=\left(p_{1}-p_{2}\right)^{2} / m-$ энергия частиц, разведенных на бесконечность, а $\rho$ - прицельный параметр. Отсюда,

$$
r_{0}=\frac{\rho^{2}}{2}+\sqrt{\frac{\rho^{4}}{4}-\frac{\alpha}{E}}
$$

и решение возможно лишь при ограничении снизу на $E: E \geqslant 4 \alpha / \rho^{4}$.

При $E=4 \alpha / b^{4}$ выражение

$$
1-\frac{b^{2}}{r^{2}}+\frac{\alpha}{r^{4} E}=\left(1-\frac{\rho^{2}}{2 r}\right)^{2}>0
$$

где $r>\rho^{2} / 2$.

2. Пусть потенциал притяжения имеет вид

$$
\begin{gathered}
\Phi(r)=-4 U_{0} \frac{\sigma^{6}}{r^{6}}, \\
E_{\min }=\min E=4 U_{0} \sigma^{6} \max _{r_{0}<\rho} \frac{1}{r_{0}^{6}}\left(\frac{\rho^{2}}{r_{0}^{2}}-1\right)^{-1}=27 U_{0} \frac{\sigma^{6}}{\rho^{6}} .
\end{gathered}
$$

Отсюда мы получаем

$$
\frac{\Phi(r)}{E_{\min }}=-\frac{4}{27} \cdot \frac{\rho^{6}}{r^{6}}
$$

Отметим, что при $E<E_{\min }$, если нет члена, отвечающего за отталкивание, возникает явление "падения на центр", т.е. схлопывание частиц.

3. Для функции

$$
\Phi=4 U_{0}\left(\frac{\sigma^{6}}{r^{6}}-\frac{\sigma^{12}}{r^{12}}\right)\left(\frac{\rho^{2}}{r^{2}}-1\right)^{-1}
$$

уравнение на точки возможного экстремума имеет вид:

$$
\Phi^{\prime}(x)=8 \varepsilon a^{6} \frac{3 x^{8}-2 \rho^{2} x^{6}-6 \sigma^{6} x^{2}+5 \sigma^{6} \rho^{2}}{x^{11}(-\rho+x)^{2}(x+\rho)^{2}}=0 \quad x=r_{0} .
$$

График $\Phi\left(r_{0}\right)$ при заданном прицельном параметре $\rho=2 \sigma$ приведен на рис. 1.

При $E$ меньшем некоторого $E_{0}$ возникает барьер глубины $E_{\max }^{\text {лок }}-E_{\min }^{\text {лок }}$, для которого при заданной температуре известны вероятности проникновения частиц в ямку [31], [32] за счет теплового шума. Это значит, что ниже этого барьера распределения, которые были получены ранее для идеального газа, неверны, поскольку существует вероятность проникновения через барьер при заданной температуре. Таким образом, максимум барьера является естественным минимумом для энергии $\left(p_{1}-p_{2}\right)^{2} / m$, ниже которого мы не имеем права использовать приведенное выше распределение. 


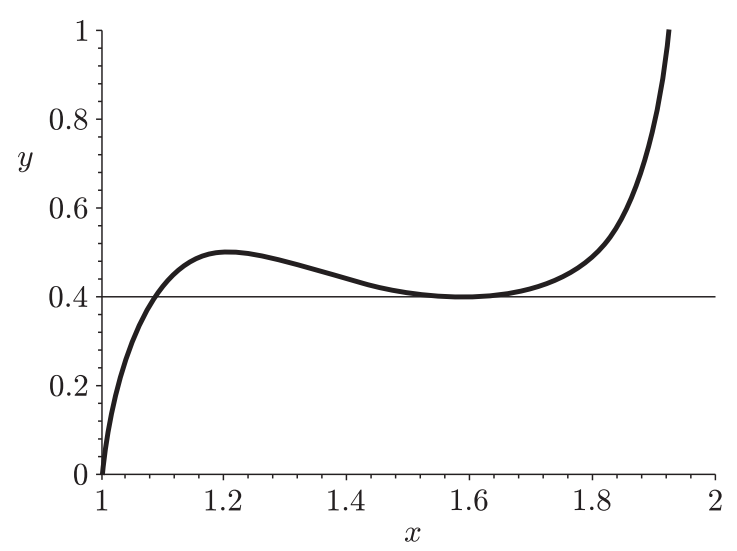

Рис. 1. Потенциальный барьер, мешающий частице, падающей из $-\infty$, проникнуть в потенциальную ямку

В работах [31], [32] вычислено, сколько времени пребывает частица при заданной температуре (тепловой шум) в ямке высоты $h$ и глубины $\delta$. Если число частиц $N \rightarrow \infty$, то это время пропорционально количеству частиц, попавших в ямку. Непонятным образом это согласуется с нашими оценками [8] попадания в конденсат и дает величину конденсата $E_{\min }(\rho)$, которая при заданном прицельном параметре $\rho$ отвечает одной и той же точке поворота $r_{0} \cong 1.21$ для потенциала Леннарда-Джонса с $n=12, r_{0}=1.02$ с $n=18$ и $r_{0}=1.28$ с $n=7$.

А так как $\rho^{3}$ для идеального газа имеет порядок $2 V_{\text {уд }} \gg \rho^{3}$, то соответственно можно определить и $E_{\min }$ из уравнения (63) при указанных $r_{0}$ и $\rho=\sqrt[3]{2 V_{\text {уд }}}$, отвечающее тем газам, взаимодействие между частицами которого лучше описывается потенциалом Леннарда-Джонса с данным $n$. Если последнее известно, то $E_{\mathrm{min}}$ определяется через $V_{\text {уд }}$.

Через $\mathscr{P}_{\rho}\left(\left(p_{1}-p_{2}\right)^{2} / m\right)$ мы определим одномерное распределение разности импульсов в задаче рассеяния [11], [33]

$$
\mathscr{P}_{\rho}\left(\frac{\left(p_{1}-p_{2}\right)^{2}}{m}\right)=\frac{1}{e^{\left(p_{1}-p_{2}\right)^{2} / m k T-\mu^{\prime} / k T}-1}\left(\int_{p_{\min }}^{\infty} \frac{d \xi}{e^{\xi^{2} / m k T-\mu^{\prime} / k T}-1}\right)^{-1} .
$$

Здесь $\rho_{\min }=\sqrt{m E_{\min }}$, а $E_{\min }$ определяется, как сказано выше, для задачи рассеяния взаимодействием вида потенциала Леннарда-Джонса.

В распределении (55) имеется химпотенциал $\mu_{1}$, который связан с химпотенциалом $\mu$ для распределения с "одетым" потенциалом следующим соотношением, которое выражает тот факт, что число частиц одномерной задачи рассеяния при фиксированном параметре рассеяния $\rho$ имеет порядок $\sqrt[3]{N / 2}$, где $N-$ полное число частиц вне конденсата.

Одетый потенциал $u$ зависит от трехмерного импульса $p$ и не зависит от координат при редукции к задаче рассеяния [13]. Поэтому химпотенциал $\mu_{1}$ связан с химпотенциалом $\mu<0$ задачи о распределении с одетым потенциалом соотношением

$$
\frac{2}{3} \pi \int \frac{p^{2} d p}{e^{\left(p^{2} / 2 m+u(p)-\mu\right) / k T}-1}=\left(\int_{p_{\min }}^{\infty} \frac{d p}{e^{p^{2} / m k T-\mu_{1} / k T}-1}\right)^{3} .
$$


Если $u(p)$ положительно, то $p_{\min }=0$, а значит, $\mu_{1}<0$.

В интегральном уравнении среднего поля (55) мы можем опустить внешний потенциал $u_{0}$, поскольку он равен нулю внутри объема, и записать окончательно [13]

$$
\begin{aligned}
u(p, \rho)=\frac{8}{9} \pi^{2} \frac{N}{V} \int_{0}^{\infty}\left[\int_{r_{0}(1 / E, \rho)}^{\infty}\right. & \frac{\Phi(r) r^{2} d r}{\sqrt{1-\rho^{2} / r^{2}-\Phi(r) / E}} \\
& \times\left[\int_{r_{0}}^{\infty}\left\{\left(\sqrt{1-\frac{\rho^{2}}{r^{2}}-\frac{\Phi(r)}{E}}\right)^{-1}-1\right\} d r\right]^{-1} \\
& \times \frac{1}{e^{\left(\left(p^{\prime}\right)^{2} / 2 m+u\left(p^{\prime}, \rho\right)-\mu\right) / k T}-1} \\
& \left.\times \frac{1}{e^{\left(\left(p-p^{\prime}\right)^{2} / m-\mu_{1}\right) / k T}-1}\left(p^{\prime}\right)^{2} d p^{\prime}\right] \\
& \times\left\{\int_{p_{\min }}^{\infty} \frac{d p}{e^{\left(p^{2} / m-\mu_{1}\right) / k T}-1}\right\}^{-4}
\end{aligned}
$$

где $V$ - объем, $r_{0}=r_{0}(\rho, E), E=\left(p-p^{\prime}\right)^{2} / m$.

Как сказано в [13],

$$
Z=\frac{1}{V_{\text {уд }}^{2 / 3}} \int_{0}^{V_{\text {уд }}^{1 / 3}} \rho d \rho \int \frac{p^{2}}{2 m} \frac{p^{2} d p}{e^{p^{2} / 2 m+u(p, \rho)-\mu / k T}-1} \cdot\left\{\int \frac{p^{2} d p}{e^{p^{2} / 2 m+u(p, \rho)-\mu / k T}-1}\right\}^{-1} .
$$

Когда задача рассеяния рассматривается во всем пространстве, то распределение

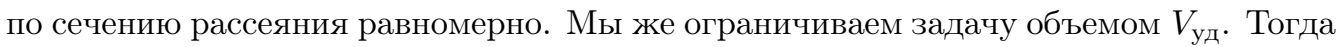
равномерность за счет границы не будет иметь места, по крайней мере вне области, где

$$
\frac{d Z}{d V_{\text {уд }}}<\frac{Z}{V_{\text {уд }}} .
$$

Распределение $\mathscr{P}(\rho)$ по $\rho$ при разных предположениях ${ }^{2}$ может быть различным (см. [34], парадокс Бертрана, [35], [36]). При этом $Z$ будет равно выражению типа $(67)$, усредненному по распределению прямых в шаре радиуса $\sqrt[3]{V_{\text {уд }}}$, отстоящих друг от друга на расстоянии $\rho$

$$
\begin{aligned}
Z=\frac{1}{V_{\text {уд }}^{2 / 3}} & \int_{0}^{V_{\text {уд }}^{1 / 3}} \mathscr{P}(\rho) \rho d \rho \int \frac{p^{2}}{2 m} \frac{p^{2} d p}{e^{p^{2} / 2 m+u(p, \rho)-\mu / k T}-1} \\
& \times\left\{\int \frac{p^{2} d p}{e^{p^{2} / 2 m+u(p, \rho)-\mu / k T}-1}\right\}^{-1} .
\end{aligned}
$$

Поэтому $Z$ можно взять в некоторой средней точке $\rho_{\text {ср }}\left(T, V_{\text {уд }}\right)$. Тогда $d Z / d V_{\text {уд }}=$ $d Z / d \rho_{\mathrm{cp}} \cdot d \rho_{\text {ср }} / d V_{\text {уд }}=-\infty$, а значит, $d Z / d \rho_{\text {ср }}=-\infty$.

Если в этой точке асимптотика при $p \rightarrow \infty$ решения $u\left(p, \rho_{\mathrm{cp}}\right)$ имеет вид $-p^{2} / 2 m+$ $\alpha\left(\rho_{\mathrm{cp}}\right)|\ln p|$, где $d \alpha / d \rho_{\mathrm{cp}}>0$, то это приводит к области, где $\frac{d Z}{d \rho_{\mathrm{cp}}}=-\infty$, определяющей $\lambda$-переход к конденсатному состоянию и закону

$$
\frac{d Z}{d P}=\frac{V_{\text {уд }}}{k T}
$$

при $P>P_{\lambda}$ и $V_{\text {уд }}>V_{\lambda}\left(P_{\lambda}\right.$ и $V_{\lambda}$ зависят от $\left.T\right)$.

\footnotetext{
${ }^{2}$ Например, А.М. Чеботарев предложил следующее распределение прицельного параметра: $\mathrm{P}(\rho \leqslant r)=1-\left(1-r^{2}\right)^{3 / 2}$.
} 
Уравнение для одетого потенциала имеет вид (мы опустили для простоты химпотенциал)

$$
u(p, \rho)=\int_{0}^{\infty} \frac{F\left((p-\eta)^{2}\right) \Theta\left((p-\eta)^{2}\right) \eta^{2} d \eta}{e^{\left(p^{2} / 2 m+u(\eta, \rho)\right) / k T}-1} \cdot\left\{\int_{p_{\min }}^{\infty} \frac{d p}{e^{p^{2} / m k T}-1}\right\}^{-4}
$$

где

$$
\begin{aligned}
F(m E)=\frac{8}{9} \frac{\pi^{2}}{\rho^{3}} \int_{r_{0}(1 / E, \rho)}^{\infty} & \frac{\Phi(r) r^{2} d r}{\sqrt{1-\rho^{2} / r^{2}-\Phi(r) / E}} \\
& \times\left[\int_{r_{0}}^{\infty}\left\{\left(\sqrt{1-\frac{\rho^{2}}{r^{2}}-\frac{\Phi(r)}{E}}\right)^{-1}-1\right\} d r\right]^{-1} \cdot \frac{1}{e^{E / k T}-1},
\end{aligned}
$$

$\Theta\left((p-\eta)^{2}\right)$ отлично от нуля лишь в области $\left|p-p^{\prime}\right|^{2} / m \geqslant E_{\min }$, а $\rho^{3}=V_{\text {уд }}$.

Перепишем это уравнение в виде

$$
u(p, \rho)=\frac{1}{2} \int_{-\infty}^{\infty} \frac{F\left((p-\eta)^{2}\right) \Theta\left((p-\eta)^{2}\right) \eta^{2} d \eta}{e^{\left(\eta^{2} / 2 m+u(\eta, \rho)\right) / k T}-1} \cdot\left\{\int_{p_{\min }}^{\infty} \frac{d p}{e^{p^{2} / m k T}-1}\right\}^{-4}
$$

и сделаем замену $(p-\eta)^{2}=\xi^{2}$.

Тогда

$$
\begin{aligned}
u(p, \rho)=\frac{1}{2}\left\{\int_{\sqrt{m E_{\min }}}^{\infty} \frac{F\left(\xi^{2}\right) d \xi}{e^{\left(\eta^{2} / 2 m+u(\eta, \rho)\right) / k T}-1}+\int_{-\infty}^{\sqrt{m E_{\min }}} \frac{F\left(\xi^{2}\right) d \xi}{e^{\left(\eta^{2} / 2 m+u(\eta, \rho)\right) / k T}-1}\right\} \\
\quad \times\left\{\int_{p_{\min }}^{\infty} \frac{d p}{e^{p^{2} / m k T}-1}\right\}^{-4} \cdot
\end{aligned}
$$

Мы будем искать условия, при которых решение этого уравнения при $p \rightarrow \infty$ имеет вид

$$
u(p)=-\frac{p^{2}}{2 m}+c(\rho)
$$

где $d c / d \rho_{\mathrm{cp}}>0$.

Прежде всего отметим, что верхний предел интеграла по $\eta$ в силу доказательств и оценок, аналогичных приведенным в теоремах, мы положили равным бесконечности, поскольку $E$ в $(10),(12),(14)$ (не путать с $E=\left(p-p^{\prime}\right)^{2} / m$ в задаче рассеяния) велико, а подынтегральное выражение быстро убывает и разность между пределом до $\sqrt{2 m E}$ и $\infty$ меньше приведенных оценок.

После указанной замены мы представляем член $\eta^{2}$ в виде $\eta^{2}=(\xi-p)^{2}$. Но интеграл от $2 p \xi$ по $\xi$ в силу того, что функция симметрична, обращается в нуль. Таким образом, мы получаем, что член в уравнении (69) справа пропорционален $p^{2}$. Теперь достаточно приравнять интерал по $\xi$ при $p \rightarrow \infty$ величине $-p^{2} / 2 m$. У нас при этом остается произвол в выборе константы $c\left(\rho_{\mathrm{cp}}\right)$.

После замены получаем

$$
u(p, \rho)=-\frac{p^{2}}{2 m}+w(p, \rho)
$$

причем при $p \rightarrow \infty$ выполнено $w(p, \rho)=c(\rho)|\ln p|$, и можно написать уравнение для функции $w(p, \rho)>0, d c\left(\rho_{\mathrm{cp}}\right) / d \rho_{\mathrm{cp}}>0$. При этом фазовый $\lambda$-переход будет, как и 


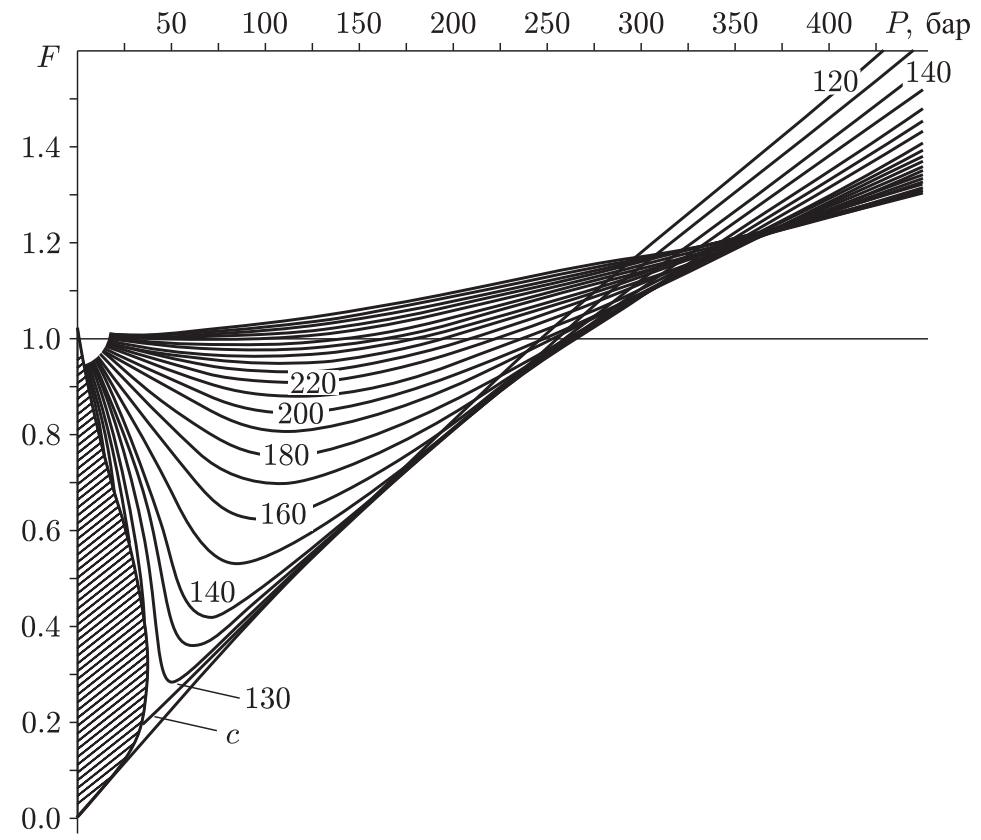

Рис. 2. Диаграмма Хоугена-Ватсона для азота; $c$ - критическая температура

минимальная точка конденсата, зависеть от степени отталкивающего члена в потенциале Леннарда-Джонса, а также от отношения $\gamma=\sigma / V_{\text {уд }}^{1 / 3}$ и от отношения $\alpha=$

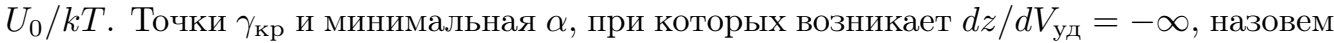

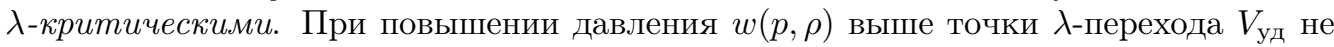
меняется. Это значит, что объем $V$ не уменьшается, но уменьшается при повышении давления, но одновременно уменьшается и число частиц вне бозе-конденсата. Возможно, все частицы перешли в димеры, и поэтому общее число частиц уменьшилось. Далее они все перешли в тримеры и т.д. Объем $V$ уменьшился, а удельный

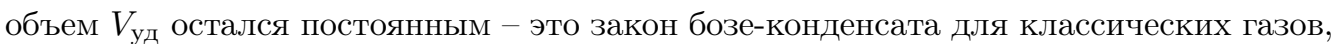
или точнее - закон образования кластеров.

Для азота при критической температуре уже при $P \geqslant 50$ атм. $V_{\text {уд }}$ перестает меняться. Для более высоких температур $\lambda$-критическое давление увеличивается; см. те участки изотерм, которые становятся наклонными прямыми, на диаграмме, приведенной на рис. 2.

При дальнейшем повышении давления газ может непрерывным образом (без скачка $\left.V_{\text {уд }}\right)$ превратиться в жидкость. Этот фазовый переход существенно отличается от фазового перехода первого рода в газе Ван дер Ваальса и других феноменологических моделях.

Приношу глубокую благодарность Д. С. Голикову за численный счет.

\section{СПИСОК ЦИТИРОВАННОЙ ЛИТЕРАТУРЫ}

[1] А. Н. Ширяев, Верояностъ, Наука, М., 1989.

[2] В. В. Козлов, Тепловое равновесие по Гиббсу и Пуанкаре, Современная математика, Ин-т компьютер. исслед., М.-Ижевск, 2002. 
[3] G. E. Andrews, The Theory of Partitions, Encyclopedia Math. Appl., 2, Addison-Wesley Publ., London, 1976.

[4] А. М. Вершик, "Статистическая механика комбинаторных разбиений и их предельные конфигурации", Функи. анализ и его прил., 30:2 (1996), 19-39.

[5] V. P. Maslov, "Quantum linguistic statistics", Russ. J. Math. Phys., 13:3 (2006), 315-325.

[6] В. П. Маслов, Т. В. Маслова, "О законе Ципфа и ранговых распределениях в лингвистике и семиотике", Матем. заметки, 80:5 (2006), 718-732.

[7] V.P. Maslov, "The Zipf-Mandelbrot law: quantization and an application to the stock market", Russ. J. Math. Phys., 12:4 (2005), 483-488.

[8] В.П. Маслов, В.Е. Назайкинский, "О распределении целочисленных случайных величин, связанных двумя линейными неравенствами. I", Матем. заметки, 83:4 (2008), $559-580$.

[9] В.П. Маслов, "Нелинейное среднее в экономике", Матем. заметки, 78:3 (2005), 377-395.

[10] V.P. Maslov, "New look on the thermodynamics of gas and at the clasretizaton", Russ. J. Math. Phys., 15:3 (2008), 494-511.

[11] В.П. Маслов, В.Е. Назайкинский, "О распределении целочисленных случайных величин, связанных одним линейным неравенством. ІІІ”, Матем. заметки, 83:6 (2008), $880-898$.

[12] В. П. Маслов, "Новая концепция процесса нуклеации", ТМФ, 156:1 (2008), 159-160.

[13] V. P. Maslov, "New theory of nucleation", Russ. J. Math. Phys., 15:3 (2008), 401-410.

[14] В.П. Маслов, П. П. Мосолов, "Асимптотическое поведение траекторий точечных зарядов, взаимодействующих по закону Кулона", Изв. АН СССР. Сер. матем., 42:5 (1978), 1063-1100.

[15] Н.Н. Боголюбов, Избранные труды. В трех томах, т. 2: К теории сверхтекучести, Наукова думка, Киев, 1970.

[16] V. P. Maslov, "On the superfluidity of classical liquid in nanotubes. I. Case of even number of neutrons", Russ. J. Math. Phys., 14:3 (2007), 304-318.

[17] V. P. Maslov, "On the superfluidity of classical liquid in nanotubes. II. Case of odd number of neutrons", Russ. J. Math. Phys., 14:4 (2007), 453-464.

[18] V.P. Maslov, "On the superfluidity of classical liquid in nanotubes. III", Russ. J. Math. Phys., 15:1 (2008), 98-101.

[19] G. Hummer, J. C. Rasaiah, J. P. Noworyta, "Water conduction through the hydrophobic channel of a carbon nanotube", Nature, 414 (2001), 188-190.

[20] S. Joseph, N. R. Aluru, "Why are carbon nanotubes fast transporters of water?", Nanoletters, 8:2 (2008), 452-458.

[21] A. Noy, H. G. Park, F. Fornasiero, J. K. Holt, C. P. Grigoropoulos, O. Bakajin, "Nanofluidics in carbon nanotubes", Nanotoday, 2:6 (2007), 22-29.

[22] A. I. Skoulidas, D. M. Ackerman, J. K. Johnson, D. S. Sholl, "Rapid transport of gases in carbon nanotubes", id. 185901, Phys. Rev. Lett., 89:18 (2002), 4 p..

[23] V.P. Maslov, "Quasi-particles associated with Lagrangian manifolds corresponding to semiclassical self-consistent fields. III", Russ. J. Math. Phys., 3:2 (1995), 271-276.

[24] В.П. Маслов, Теория возмущений и асимптотические методы, Изд-во МГУ, М., 1965.

[25] В. П. Маслов, "О фазовом переходе для классических фермионов", Матем. заметки, 63:4 (1998), 635-637.

[26] В. П. Маслов, "О фазовом переходе для классических бозонов, фермионов и объемных классических частиц", Матем. заметки, 63:5 (1998), 792-795.

[27] В. П. Маслов, “Асимптотика при $N \rightarrow \infty$ для $N$ классических фермионов и бозонов", Матем. заметки, 66:6 (1999), 849-866.

[28] В.П. Маслов, "Учет взаимодействия между частицами в новой теории нуклеации, квазичастицы, квантование вихрей и двухчастичеая функция распределения", $M a$ тем. заметки, 83:6 (2008), 864-879. 
[29] В. Б. Берестецкий, Е. М. Лифшиц, Л. П. Питаевский, Теоретическая физика. Т. 4. Квантовая электродинамика, Наука, М., 1980.

[30] А. И. Бурштейн, Молекулярная физика, Наука, Новосибирск, 1986.

[31] Л. С. Понтрягин, А. А. Андронов, А. А. Витт, "О статистическом рассмотрении динамических систем”, ЖЭТФ, 3:3 (1933), 165-180.

[32] P.S. Landa, "Theory of fluctuational transitions and its applications", J. Comm. Tech. Electron., 46:10 (2001), 1069-1107.

[33] В.П. Маслов, В.Е. Назайкинский, "О распределении целочисленных случайных величин, связанных двумя линейными соотношениями”, Матем. заметки, 84:1 (2008), 69-98.

[34] J. Bertrand, Calcul des probabilites, Gauthier-Villars, Paris, 1889.

[35] Р. В. Амбарцумян, Й. Мекке, Д. Штойян, Введение в стохастическую геометрию, М., Наука, 1989.

[36] D. A. Klain, G.-C. Rota, Introduction to Geometric Probability, Lezioni Lincee, Cambridge Univ. Press, Cambridge, 1997.

В. П. Маслов

Московский государственный университет

им. М. В. Ломоносова

E-mail:v.p.maslov@mail.ru
Поступило 16.10.2008 